\title{
SUBNORMAL OPERATORS, TOEPLITZ OPERATORS AND SPECTRAL INCLUSION ${ }^{1}$
}

\author{
BY
}

\author{
GERARD E. KEOUGH
}

\begin{abstract}
Let $S$ be a subnormal operator on the Hilbert space $H$, and let $N=\int z d E(z)$ be its minimal normal extension on $K$. Let $\mu$ be a scalar spectral measure for $N$. If $f \in L^{\infty}(\mu)$, define $T_{f}=\left.P f(N)\right|_{H}: H \rightarrow H$, where $P: K \rightarrow H$ denotes orthogonal projection. $S$ has the $C^{*}$-Spectral Inclusion Property ( $C^{*}$-SIP) if $\sigma(f(N)) \subseteq \sigma\left(T_{f}\right)$, for all $f \in C(\sigma(N))$, and $S$ has the $W^{*}$-Spectral Inclusion Property ( $W^{*}$-SIP) if $\sigma(f(N)) \subseteq \sigma\left(T_{f}\right)$, for all $f \in L^{\infty}(\mu)$.

It is shown that $S$ has the $C^{*}$-SIP if and only if $\sigma(N)=\Pi(S)$, the approximate point spectrum of $S$. This is equivalent to requiring that $E(\Delta) K$ have angle 0 with $H$, for all nonempty, relatively open $\Delta \subseteq \sigma(N)$. $S$ has the $W^{*}$-SIP if this angle condition holds for all proper Borel subsets of $\sigma(N)$.

If $S$ is pure and has the $C^{*}$ - or $W^{*}$-SIP, then it is shown that $\sigma(f(N)) \subseteq \sigma_{e}\left(T_{f}\right)$, for all appropriate $f$.
\end{abstract}

The spectrum of an operator on a Hilbert space has always been a valuable tool for the operator theorist. In particular, the study of normal operators is quite complete (see, for example, Halmos [1951]). Two classes of operators where knowledge of the spectrum and/or spectral properties has been crucial are the class of Toeplitz operators and the class of subnormal operators. In the former class, a result of Hartman and Wintner states that the spectrum of every Toeplitz operator contains that of its associated Laurent operator (Hartman-Wintner [1950]). In the latter class, a theorem obtained by Halmos states that the spectrum of a subnormal operator contains that of its minimal normal extension (Halmos [1952]). A recent question of Halmos is to describe, if possible, what the relationship is between these two results-perhaps one may even be a consequence of the other?

To formulate a setting in which to view the two results, we shall adopt the point of view that Toeplitz operators are, in a reasonable sense, generated by a particular subnormal operator-namely, the unilateral shift (i.e., multiplication by the independent variable on the usual Hardy space). To see what this means, consider that the bilateral shift is the minimal normal extension of the unilateral shift, and that the classical Laurent operators form exactly the von Neumann algebra generated by the bilateral shift. Toeplitz operators are then compressions of operators in some

Received by the editors June 22, 1979 and, in revised form, January 11, 1980.

AMS (MOS) subject classifications (1970). Primary 47B20, 47A10; Secondary 47B35, 47A20.

Key words and phrases. Subnormal operator, spectral inclusion theorems, angle between subspaces, reducing essential spectrum, extremely noncompact operator.

${ }^{1}$ These results constitute a portion of the author's Ph.D. disseration, written under the direction of Professor John B. Conway, at Indiana University, to whom the author is deeply indebted for his enthusiastic encouragement. 
algebra (here, the von Neumann algebra) generated by the minimal normal extension of a subnormal operator (here, the unilateral shift). In general, we shall investigate what relationship should exist between a subnormal operator and its minimal normal extension so that the spectrum of every operator in a particular algebra generated by this normal operator will be contained inside that of its compression to the space upon which the subnormal operator acts.

$\S 1$ of this paper will establish the notation used throughout the remainder, as well as the definition of the Spectral Inclusion Property. $\$ 2$ contains the theorem which characterizes those subnormal operators for which the following is true: every operator in the $C^{*}$-algebra generated by its minimal normal extension has spectrum contained inside that of its compression to the space upon which the subnormal operator acts. $\$ 3$ contains an analogous characterization of those subnormal operators for which this same result holds for all operators in the von Neumann algebra generated by the minimal normal extension. Both $\S \S 2$ and 3 provide examples of the application of each theorem. $\$ 4$ will examine the results of the previous sections more carefully when the assumptions of purity and essential normality are placed on a subnormal operator.

Throughout this paper, all Hilbert spaces are assumed to be complex, separable, and infinite dimensional, having an inner product which is generically denoted by $\langle\cdot, \cdot\rangle$. The term operator will always mean a bounded linear transformation; projection will mean an idempotent, selfadjoint operator; and measure will mean a positive Borel measure on the complex plane, which is denoted by $\mathbf{C}$.

1. Notation and definitions. Let $S$ denote a fixed, but arbitrary, subnormal operator acting on a Hilbert space $H$. By definition, this means that there is a Hilbert space $K$ containing $H$, and a normal operator $N$ acting on $K$, such that $N H \subseteq H$, and $S=\left.N\right|_{H}$ (the restriction of $N$ to $H$ ). $N$ is said to be a normal extension for $S$. We shall, in fact, assume that $N$ is a minimal normal extension for $S$-i.e., that if $K_{1}$ is any subspace of $K$ which contains $H$ and reduces $N$, then necessarily $K_{1}=K$. Let $P: K \rightarrow H$ denote projection.

Let $E$ denote the spectral measure for $N$, and let $\mu$ be a measure defined on $\sigma(N)$, the spectrum of $N$, such that $\mu(\Delta)=0$ if and only if $E(\Delta)$ is the zero operator, whenever $\Delta \subseteq \sigma(N)$ is any Borel set (such a measure $\mu$ is called a scalar spectral measure for $N)$. The mapping $f \rightarrow f(N)$, where $f(N)$ is defined via the spectral integral $f(N)=\int f(z) d E(z)$, produces a *-isometric isomorphism of $L^{\infty}(\mu)$ onto $W^{*}(N)$, the von Neumann algebra generated by $N$, which is a homeomorphism when $L^{\infty}(\mu)$ is given the weak-* topology which it inherits as the dual space of $L^{1}(\mu)$, and $W^{*}(N)$ is given the weak operator topology. In particular, if $C(\sigma(N))$ denotes the space of continuous, complex-valued functions defined on $\sigma(N)$, then the image of $C(\sigma(N))$ under this mapping is exactly $C^{*}(N)$, the $C^{*}$-algebra generated by $N$ and the identity operator. (See for example, Douglas [1972, p. 112].)

For each function $f \in L^{\infty}(\mu)$, define an operator $T_{f}$ on $H$ via compression: i.e., $T_{f} h=P f(N) h$, for all $h \in H$. The mapping $f \rightarrow T_{f}$ is linear and contractive, and respects the adjoint operation. When $S$ is the unilateral shift, the mapping $f \rightarrow T_{f}$ is 
well understood, since the operators $\left\{T_{f} \mid f\right.$ is in $\left.L^{\infty}(\mu)\right\}$ are exactly the Toeplitz operators. The Hartman-Wintner Theorem is the statement that $\sigma(f(N)) \subseteq \sigma\left(T_{f}\right)$, for all $f \in L^{\infty}(\mu)$. As a corollary of this result, it follows that the mapping $f \rightarrow T_{f}$ is an isometry, when $S$ is the unilateral shift (cf. Douglas [1972, Chapter 7]).

Motivated by this situation, we shall say that a subnormal operator $S$ has the $C^{*}$-Spectral Inclusion Property (abbreviated $C^{*}$-SIP) if $\sigma(f(N)) \subseteq \sigma\left(T_{f}\right)$, for all $f \in C(\sigma(N))$. Additionally, $S$ has the $W^{*}$-Spectral Inclusion Property (abbreviated $W^{*}$-SIP) if this containment holds for all $f \in L^{\infty}(\mu)$. For example, the unilateral shift has the $W^{*}$-SIP.

Before continuing, it should be recorded that the $C^{*}$-SIP (respectively $W^{*}$-SIP) is a unitary invariant of a subnormal operator $S$. For $i=1,2$, let $S_{i}$ be a subnormal operator on the Hilbert space $H_{i}$, and let $N_{i}$ be its minimal normal extension on $K_{i}$. If $U: H_{1} \rightarrow H_{2}$ is a unitary transformation such that $U S_{1}=S_{2} U$, then there exists a unitary transformation $V: K_{1} \rightarrow K_{2}$ with the property that $V N_{1}=N_{2} V$, and such that $V H_{1}=H_{2}$ and $V h=U h$, for all $h \in H_{1}$ (Halmos [1967, Problem 155]). Since $V C^{*}\left(N_{1}\right)=C^{*}\left(N_{2}\right)$ and $V W^{*}\left(N_{1}\right)=W^{*}\left(N_{2}\right)$, it follows that $S_{1}$ has the $C^{*}$-SIP (resp. $W^{*}$-SIP) if and only if $S_{2}$ has the $C^{*}$-SIP (resp. $W^{*}$-SIP).

Even if the subnormal operator $S$ does not have the $C^{*}$ - or $W^{*}$-SIP, the following lemma is of use.

1.1. Lemma. $\left\{f \in L^{\infty}(\mu) \mid \sigma(f(N)) \subseteq \sigma\left(T_{f}\right)\right\}$ is norm closed in $L^{\infty}(\mu)$.

Proof. Suppose that $\left\{f_{n}\right\} \subseteq L^{\infty}(\mu)$ is a sequence of functions such that, for all indices $n, \sigma\left(f_{n}(N)\right) \subseteq \sigma\left(T_{f_{n}}\right)$, and suppose that $f_{n} \rightarrow f \in L^{\infty}(\mu)$ in norm. To establish that $\sigma(f(N)) \subseteq \sigma\left(T_{f}\right)$, it is enough to show that if $T_{f}$ is invertible, then $f$ is invertible in $L^{\infty}(\mu)$.

Suppose, then, that $0 \notin \sigma\left(T_{f}\right)$, and choose $\varepsilon>0$ such that $3 \varepsilon<\operatorname{dist}\left(0, \sigma\left(T_{f}\right)\right)$. Let $O=\left\{z \mid \operatorname{dist}\left(z, \sigma\left(T_{f}\right)\right)<\varepsilon\right\}$. Because the spectrum is upper semicontinuous (Halmos [1967, Problem 86]), there exists $\delta>0$ such that, whenever $A$ is an operator on $H$ which satisfies $\left\|A-T_{f}\right\|<\delta$, then $\sigma(A) \subseteq O$. By assumption, it follows that $\sigma\left(T_{f_{n}}\right) \subseteq \varnothing$, whenever the index $n$ is sufficiently large.

Suppose that $f$ is not invertible. Let $\Delta=f^{-1}(\{z:|z|<\varepsilon\})$. There is no loss in generality by supposing that $\varepsilon$ has already been chosen to be small enough so that $0<\mu(\Delta)<\mu(\sigma(N))$. Thus, $\varepsilon \cdot \mu(\Delta)>\int_{\Delta}|f| d \mu \geqslant \int_{\Delta}\left(\left|f_{n}\right|-\varepsilon\right) d \mu \geqslant \varepsilon \cdot \mu(\Delta)$, whenever $n$ is a large index-which is a contradiction. Thus, $\mu(\Delta)=0$, and $f$ is invertible.

Lastly, we wish to recall that if $M_{1}$ and $M_{2}$ are two subspaces of $K$, then the angle between $M_{1}$ and $M_{2}$, denoted $\theta\left(M_{1}, M_{2}\right)$, is defined to be that real number in $[0, \pi / 2]$ such that $\cos \theta\left(M_{1}, M_{2}\right)=\sup \left\{\left|\left\langle m_{1}, m_{2}\right\rangle\right|: m_{1} \in M_{1}, m_{2} \in M_{2}\right.$, and $\left.\left\|m_{1}\right\|=\left\|m_{2}\right\|=1\right\}$. If, for $i=1,2, Q_{i}$ denotes the projection of $K$ onto $M_{i}$, then $\left\|\left.Q_{2}\right|_{M_{1}}\right\|=\left\|\left.Q_{1}\right|_{M_{2}}\right\|=\cos \theta\left(M_{1}, M_{2}\right)$, and $\left\|\left.Q_{2} Q_{1}\right|_{M_{2}}\right\|=\left\|\left.Q_{1} Q_{2}\right|_{M_{1}}\right\|=$ $\cos ^{2} \theta\left(M_{1}, M_{2}\right)$ (for example, consult Halmos [1951, §15]).

2. The $C^{*}$-SIP. We shall continue to maintain the notation that if $S$ denotes a subnormal operator on $H$, then $N=\int z d E(z)$ is its minimal normal extension on $K, \mu$ is a scalar spectral measure for $N$, and $P: K \rightarrow H$ is projection. 
Before giving the characterization of the $C^{*}$-SIP, we make the following definition. If $L$ is an operator on $K$, then the $H$-approximate point spectrum of $L$, denoted $\Pi_{H}(L)$, consists of those scalars $\lambda \in \sigma(L)$ for which there exists a sequence of unit vectors $\left\{h_{n}\right\} \subseteq H$ such that $\left\|(L-\lambda) h_{n}\right\| \rightarrow 0$. If $f \in L^{\infty}(\mu)$, then $\Pi_{H}(f(N))$ is a closed subset of the approximate point spectrum of $T_{f}$, which will be denoted by $\Pi\left(T_{f}\right)$.

2.1. THEOREM. Given a subnormal operator $S$, the following are logically equivalent statements:

(i) $S$ has the $C^{*}-S I P$;

(ii) $\left\|T_{f}\right\|=\|f\|_{\infty}$, for all $f \in C\left(\sigma(N)\right.$ ) (i.e., the map $f \rightarrow T_{f}$ is an isometry on $C(\sigma(N)))$;

(iii) $\left\|T_{\chi}\right\|=1$, whenever $\chi=\chi_{\Delta}$ is the characteristic function of a nonempty, relatively open set $\Delta \subseteq \sigma(N)$;

(iv) $\theta(E(\Delta) K, H)=0$, whenever $\Delta \subseteq \sigma(N)$ is relatively open and nonempty;

(v) $\sigma(f(N))=\Pi_{H}(f(N))$, for all $f \in C(\sigma(N))$;

(vi) $\sigma(N)=\Pi(S)$.

Proof. If $S$ has the $C^{*}$-SIP, and if $f \in C(\sigma(N))$, then

$$
\|f(N)\|=\sup \{|\lambda|: \lambda \in \sigma(f(N))\} \leqslant \sup \left\{|\lambda|: \lambda \in \sigma\left(T_{f}\right)\right\} \leqslant\left\|T_{f}\right\| \leqslant\|f(N)\|,
$$

so that equality holds throughout. Thus, $\left\|T_{f}\right\|=\|f\|_{\infty}$, by use of the functional calculus.

Let $\Delta \subseteq \sigma(N)$ be relatively open and nonempty. Choose $f \in C(\sigma(N))$ such that $\|f\|_{\infty}=1$, and such that $\{z: f(z) \neq 0\} \subseteq \Delta$. Thus, $1=\left\|T_{f}\right\|=\left\|\left.P f(N)\right|_{H}\right\|=$ $\left\|\left.P f(N) E(\Delta)\right|_{H}\right\|$, and so $\left\|\left.E(\Delta)\right|_{H}\right\|=1$. This implies condition (iv), which, in general, is equivalent to condition (iii).

Suppose $\theta(E(\Delta) K, H)=0$, for all nonempty, relatively open $\Delta \subseteq \sigma(N)$. Let $f \in C(\sigma(N))$, and choose $\lambda \in \sigma(f(N))$. Let $\left\{\Delta_{n}\right\}$ be a decreasing sequence of relatively open subsets of $\sigma(f(N))$ such that $\lambda \in \cap \Delta_{n}$ and such that diam $\Delta_{n} \rightarrow 0$. $\left\{f^{-1}\left(\Delta_{n}\right)\right\}$ is a sequence of relatively open subsets of $\sigma(N)$; so there exists a sequence of unit vectors $\left\{h_{n}\right\} \subseteq H$ for which $\left\|E\left(f^{-1}\left(\Delta_{n}\right)\right) h_{n}\right\| \rightarrow 1$. It follows that

$$
\begin{aligned}
\left\|(f(N)-\lambda) h_{n}\right\|^{2}= & \int_{f^{-1}\left(\Delta_{n}\right)}|f(z)-\lambda|^{2} d\left(E(z) h_{n}, h_{n}\right) \\
& +\int_{\sigma(N) \backslash f^{-1}\left(\Delta_{n}\right)}|f(z)-\lambda|^{2} d\left(E(z) h_{n}, h_{n}\right) \\
\leqslant & \left(\operatorname{diam} \Delta_{n}\right)^{2}+4\|f\|_{\infty}^{2} \cdot\left\|\left(1-E\left(f^{-1}\left(\Delta_{n}\right)\right)\right) h_{n}\right\| .
\end{aligned}
$$

Thus, $\lambda \in \Pi_{H}(f(N))$, and $\Pi_{H}(f(N))=\sigma(f(N))$. In particular, note that $\sigma(N)=$ $\Pi_{H}(N)=\Pi(S)$, because $N$ is a normal extension for $S$.

To complete the proof, assume that $\sigma(N)=\Pi(S)$, and let

$$
p=p(\bar{z}, z)=\sum_{k=0}^{m}\left(\sum_{\substack{i+j=k \\ i, j>0}} \alpha_{i j} \bar{z}^{i} z^{j}\right)
$$


be any polynomial in the two complex variables $\bar{z}$ and $z$. It is easy to see that

$$
T_{p}=\sum_{k=0}^{m}\left(\sum_{\substack{i+j=k \\ i, j>0}} \alpha_{i j} S^{* i} S^{j}\right) .
$$

Via the functional calculus, $\sigma\left(p\left(N^{*}, N\right)\right)=\{p(\bar{\lambda}, \lambda): \lambda \in \sigma(N)\}=\{p(\bar{\lambda}, \lambda): \lambda \in$ $\Pi(S)\}$; thus to show that $\sigma\left(p\left(N^{*}, N\right)\right) \subseteq \sigma\left(T_{p}\right)$, it suffices to show that $\{p(\bar{\lambda}, \lambda)$ : $\lambda \in \Pi(S)\} \subseteq \sigma\left(T_{p}\right)$. However,

$$
T_{p}-p(\bar{\lambda}, \lambda)=\sum_{k=0}^{m}\left(\sum_{\substack{i+j=k \\ i, j>0}} \alpha_{i j}\left(S^{* i} S^{j}-\bar{\lambda}^{i} \lambda^{j}\right)\right) .
$$

If $\lambda \in \Pi(S)$, then all terms in the displayed sum fail to be bounded below, since for any $i, j \geqslant 0$ we may write

$$
S^{* i} S^{j}-\bar{\lambda}^{i} \lambda^{j}=S^{* i} q_{1}(S)(S-\lambda)+\lambda^{j} q_{2}\left(S^{*}\right)\left(S^{*}-\bar{\lambda}\right)
$$

for appropriate polynomials $q_{1}$ and $q_{2}$ (this relies on the fact that $S$ is hyponormal). Thus, $\sigma\left(p\left(N^{*}, N\right)\right) \subseteq \sigma\left(T_{p}\right)$, for all such polynomials $p$. Lemma 1.1 now implies that $S$ has the $C^{*}$-SIP.

We remark that some authors refer to $\sigma(N)$ as the "normal spectrum" of the subnormal operator $S$, and denote it as $\sigma_{\perp}(S)$. Using this terminology, Theorem 2.1 is the statement that $S$ has the $C^{*}$-SIP if, and only if, its normal spectrum and approximate point spectrum agree. For example, Abrahamse and Douglas have constructed and completely classified a class of subnormal operators called "bundle shifts" whose definition includes the condition that $\sigma_{\perp}(S)$ be contained inside $\partial \sigma(S)$, the boundary of the spectrum of $S$. This guarantees that all bundle shifts have the $C^{*}$-SIP (Abrahamse-Douglas [1976]). More recently, Bunce and Deddens have given an intrinsic characterization of $\sigma_{\perp}(S)$ for an arbitrary subnormal operator $S$, and this might be incorporated into Theorem 2.1 to eliminate the normal operator $N$ from its statement (Bunce-Deddens [1977]).

Now, let $\nu$ be any compactly supported measure on $\mathbf{C}$, and let $H^{2}(\nu)$ denote the $L^{2}$-closure of the polynomials in $L^{2}(\nu)$. Define an operator $U_{\nu}: L^{2}(\nu) \rightarrow L^{2}(\nu)$ by the equation $\left(U_{\nu} f\right)(z)=z f(z)$, for all $f \in L^{2}(\nu)$. Then $U_{\nu} H^{2}(\nu) \subseteq H^{2}(\nu)$, so let $U_{\nu}^{+}=\left.U_{\nu}\right|_{H^{2}(\nu)} \cdot U_{\nu}^{+}$is then subnormal and, by virtue of the Stone-Weierstrass Theorem, has $U_{\nu}$ as its minimal normal extension. In particular, if $m$ denotes normalized Lebesgue measure on $\partial \mathbf{D}$, the boundary of the open unit disc $\mathbf{D}=\{z$ : $|z|<1\}$, then $H^{2}(m)$ is the usual Hardy space, and $U_{m}^{+}$is the unilateral shift.

Suppose that the subnormal operator $S$ is a weighted shift with unit norm-i.e., that there is an orthonormal basis $\left\{e_{0}, e_{1}, \ldots\right\}$ for $H$ and an associated sequence of scalars $\left\{\alpha_{0}, \alpha_{1}, \ldots\right\}$ such that $\sup \left\{\left|\alpha_{n}\right|\right\}=\lim \left|\alpha_{n}\right|=1$, and $S e_{k}=\alpha_{k} e_{k+1}$, for $k=0,1, \ldots$ A theorem of Berger (see Halmos [1970]) shows that there exists a normalized measure $\hat{v}$ on $[0,1]$ such that

$$
\left|\alpha_{0} \cdots \alpha_{n-1}\right|^{2}=\int_{0}^{1} t^{2 n} d \hat{\nu}(t), \quad n \geqslant 1 .
$$

Further, if $\nu$ is defined on D by setting $d \nu\left(r e^{i \theta}\right)=d \hat{\nu}(r) d \theta / 2 \pi$, then $S$ is unitarily equivalent to $U_{\nu}^{+}$. 
2.2. Proposition. Let $S$ be a subnormal weighted shift having unit norm and trivial kernel. Then $S$ has the $C^{*}-S I P$ if and only if $S$ is unitarily equivalent to the unilateral shift $U_{m}^{+}$.

Proof. Using the characterization of Berger, Theorem 2.1 implies that $S$ has the $C^{*}$-SIP if and only if the support of the associated measure $\nu$ is the same as $\Pi(S)$. However, $\Pi(S)=\partial D$ (see, for example, Shields [1974]). It follows that $\nu=m$.

We shall close this section with an illustrative example; however, we shall need the following result:

2.3. Proposition. Let $\left\{S_{i}\right\}$ be subnormal operators such that each $S_{i}$ has the $C^{*}$-SIP. Assume that $\sup \left\{\left\|S_{i}\right\|\right\}<+\infty$. Then the direct sum $S=\Sigma \oplus S_{i}$ also has the $C^{*}$-SIP. In particular, unilateral shifts of arbitrary multiplicity have the $C^{*}-S I P$.

Proof. If, for each $i, N_{i}$ is the minimal normal extension of $S_{i}$, then $N=\Sigma \bigoplus N_{i}$ is the minimal normal extension of $S$. By assumption, $\sigma\left(N_{i}\right)=\Pi\left(S_{i}\right)$, for each index $i$; it follows that

$$
\sigma(N)=\left[\cup \sigma\left(N_{i}\right)\right]^{-}=\left[\cup \Pi\left(S_{i}\right)\right]^{-}=\Pi(S),
$$

and so $S$ has the $C^{*}$-SIP, by Theorem 2.1 .

2.4. ExAmple. Let $\nu$ be the measure defined on $\partial \mathbf{D} \cup\{0\}$ by setting $\left.\nu\right|_{\partial \mathbf{D}}=m$ and $\nu(\{0\})=1$. The operator $U_{\nu}^{+}$is subnormal, but does not have the $C^{*}$-SIP. (It is, in fact, a weighted shift, so that Proposition 2.2 applies. This particular operator was originally employed by Sarason, concerning a similarity problem for subnormal operators-see Halmos [1967], Problem 156.) In particular, let $\chi=\chi_{\{0\}}$ denote the characteristic function of $\{0\}$; then $\chi \in C\left(\sigma\left(U_{v}\right)\right)$, and an easy calculation shows that $T_{\chi}$ is a diagonal operator for which $\sigma\left(T_{\chi}\right)=\left\{0, \frac{1}{2}\right\}$ and $\left\|T_{\chi}\right\|=\frac{1}{2}$ (equivalently, $\theta\left(\right.$ kernel $\left.\left.U_{\nu}, H^{2}(\nu)\right)=\pi / 4\right)$. Now, let $\left\{\gamma_{n}\right\}$ be a sequence of positive scalars such that $\gamma_{n} \rightarrow 0$, and let $S=\sum_{n=1}^{\infty} \oplus \gamma_{n} U_{\nu}^{+}$. Then $N=\sum_{n=1}^{\infty} \oplus \gamma_{n} U_{\nu}$ is the minimal normal extension of $S$ and

$$
\sigma(N)=\left[\bigcup_{n=1}^{\infty}\left\{z:|z|=\gamma_{n}\right\}\right]^{-}=\Pi(S) .
$$

Thus, $S$ has the $C^{*}$-SIP, even though none of its factors has the property. Lastly, to anticipate the discussion of the next section, note that $\chi=\chi_{\{0\}} \notin C(\sigma(N))$, and that $\left\|T_{\chi}\right\|=\frac{1}{2}$. In particular, $\{0\}$ is not relatively open in $\sigma(N)$.

3. The $W^{*}$-SIP. We shall say that a Borel set $\Delta \subseteq \sigma(N)$ is proper if $0<E(\Delta)<$ 1. The characterization of the $W^{*}$-SIP given below parallels that of the $C^{*}$-SIP; its proof, however, will not be given in light of its similarity to the proof of Theorem 2.1.

3.1. TheOREM. Given a subnormal operator $S$, the following are logically equivalent statements:

(i) $S$ has the $W^{*}-S I P$;

(ii) $\left\|T_{f}\right\|=\|f\|_{\infty}$, for all $f \in L^{\infty}(\mu)$;

(iii) $\theta(E(\Delta) K, H)=0$, for all proper Borel sets $\Delta \subseteq \sigma(N)$;

(iv) $\sigma(f(N))=\Pi_{H}(f(N))$, for all $f \in L^{\infty}(\mu)$. 
It is important to note that the condition $\sigma(N)=\Pi(S)$ is not sufficient for the $W^{*}$-SIP. Indeed, the example given at the close of $\$ 2$ constructed a subnormal operator $S$ with the $C^{*}$-SIP for which $\theta(\operatorname{kernel} N, H)=\pi / 4$.

As an application of the theorem, we shall incorporate an earlier generalization of Toeplitz operators, due to R. G. Douglas and C. Pearcy, into the current framework (Douglas-Pearcy [1965]). The central concept in this situation is an appropriate version of the F. and M. Riesz Theorem. Adopting the terminology of the Douglas-Pearcy work, we shall say that a subspace $M \subseteq K$ is a weak Riesz subspace for $W^{*}(N)$ if, for some $L \in W^{*}(N), L k=0$ for a nonzero vector $k \in M$ implies that $L=0$. To justify the terminology, it is easy to see that this is equivalent to requiring that $E(\Delta) K \cap M$ be the zero subspace, for all proper Borel sets $\Delta \subseteq \sigma(N)$.

3.2. Proposition. If $N$ has no eigenvalues, and if $H^{\perp}$ is a weak Riesz subspace for $W^{*}(N)$, then $S$ has the $W^{*}-S I P$.

Proof. We shall merely sketch the proof, as this is mainly the proof of Theorem 1 of the article by Douglas and Pearcy. If $\Delta \subseteq \sigma(N)$ is any proper Borel set, then choose a Borel set $\Delta_{1} \subseteq \sigma(N)$ such that $\Delta \subseteq \Delta_{1}$ and such that $E(\Delta)<E\left(\Delta_{1}\right)<1$. Let $k \in K$ be a unit vector such that $k=E\left(\Delta_{1}\right) k=(1-E(\Delta)) k$. Because $H^{\perp}$ is a weak Riesz subspace for $W^{*}(N)$, it follows that $\left[E\left(\Delta_{1}\right) H\right]^{-}=E\left(\Delta_{1}\right) K$; thus, there exists a sequence of vectors $\left\{h_{n}\right\} \subseteq H$ such that $\left\|E\left(\Delta_{1}\right) h_{n}-k\right\| \rightarrow 0$. Necessarily, the sequence $\left\{\left\|h_{n}\right\|\right\}$ is bounded away from 0 , for large indices $n$. If $\chi=\chi_{\Delta}$ is the characteristic function of $\Delta$, then $\left\|T_{\chi} h_{n}\right\|<\left\|E(\Delta) h_{n}\right\| \rightarrow 0$, so that $0 \in \sigma\left(T_{\chi}\right)$. $\Delta$ was arbitrary, so that by considering complements, it follows that $1 \in \sigma\left(T_{\chi}\right)$, whenever $\chi=\chi_{\Delta}$ is the characteristic function of a proper Borel set $\Delta \subseteq \sigma(N)$. This is equivalent to the condition that $\theta(E(\Delta) K, H)=0$, for all proper Borel sets $\Delta \subseteq \sigma(N)$; thus, $S$ has the $W^{*}$-SIP.

Before giving an example of the use of the theorem, note that $H^{\perp}$ is an invariant subspace for $N^{*}$. Thus $\left.N^{*}\right|_{H^{+}}$is a subnormal operator, having $N^{*}$ as a normal extension. If the subnormal operator $S$ is pure-that is, if $S$ has no nontrivial normal summand-then $N^{*}$ is the minimal normal extension of $\left.N^{*}\right|_{H^{\perp}}$ (see Olin [1976, Lemma 5.3]); in this case, $\left.N^{*}\right|_{H^{+}}$is called the dual of $S$. Whereas Proposition 2.2 indicated that the class of pure subnormal weighted shifts was somewhat lean with respect to the $C^{*}$-SIP, the following shows that just the opposite is true for the duals of this class.

3.3. Proposition. Let $S$ be a pure, subnormal weighted shift for which $N$, its minimal normal extension, has trivial kernel. Then the dual of $S$ has the $W^{*}$-SIP. In particular, $U_{m}^{+}$(the unilateral shift) has the $W^{*}-S I P$.

Proof. We may assume $\|S\|=1$. As before, $S$ may be taken to be the operator $U_{\nu}^{+}$on $H^{2}(\nu)$, where $d \nu\left(r e^{i \theta}\right)=d \hat{\nu}(r) d \theta / 2 \pi$, for some measure $\hat{\nu}$ on $[0,1]$. A function $f \in L^{2}(\nu)$ belongs to $H^{2}(\nu)$ if and only if $f$ is analytic in $\mathbf{D}$, and its Taylor coefficients in the expansion $f(z)=\sum_{n=0}^{\infty} a_{n} z^{n}$ satisfy the relation $\sum_{n=0}^{\infty}\left|a_{n}\right|^{2} \beta_{n}<$ $+\infty$-where the scalars $\left\{\beta_{n}\right\}$ are defined as $\beta_{n}=\int_{0}^{1} t^{2 n} d \hat{\nu}(t)$ (see Frankfurt [1975]). By assumption, the measure $\nu$ has no atoms. The usual theory of analytic 
functions on $\mathbf{D}$ then implies that if $\nu(\Delta)>0$ and if $f(z)=0$, for all $z \in \Delta$ and for some $f \in H^{2}(\nu)$, then $f \equiv 0$. It follows that $H^{2}(\nu)$ is a weak Riesz subspace for $W^{*}\left(U_{\nu}^{*}\right)=W^{*}\left(U_{\nu}\right)$, so that $\left.U_{\nu}^{*}\right|_{H^{2}(\nu)^{\perp}}$ has the $W^{*}$-SIP.

Lastly, note that the dual of $U_{m}^{+}$is (unitarily equivalent to) $U_{m}^{+}$, so that $U_{m}^{+}$has the $W^{*}$-SIP.

3.4. Proposition. If $\left\{S_{i}\right\}$ are subnormal operators such that, for each index $i, S_{i}$ has the $W^{*}-S I P$, and if $\sup \left\{\left\|S_{i}\right\|\right\}<\infty$, then the direct sum $S=\Sigma \bigoplus S_{i}$ has the $W^{*}$-SIP. In particular, unilateral shifts of arbitrary multiplicity have the $W^{*}$-SIP.

Proof. If $N_{i}$ is the minimal normal extension of $S_{i}$, for each index $i$, then $N=\Sigma \oplus N_{i}$ is the minimal normal extension for $S$. Further, if $E_{i}$ is the spectral measure for $N_{i}$, then $E=\Sigma \oplus E_{i}$ is the spectral measure for $N$. If $\Delta \subseteq \sigma(N)$ is a proper Borel set, then $\Delta \cap \sigma\left(N_{i}\right)$ is either a proper Borel subset of $\sigma\left(N_{i}\right)$, or all of $\sigma\left(N_{i}\right)$, for some index $i$. Thus, we have $\theta(E(\Delta) K, H)<\theta\left(E_{i}(\Delta) K, H\right)=0$. Theorem 3.1 implies that $S$ has the $W$-SIP.

3.5. EXAMPLE. For each $i=1,2, \ldots$, let $\nu$ be the measure on $\partial \mathbf{D} \cup\{0\}$ which is defined by setting $\left.\nu_{i}\right|_{\partial \mathbf{D}}=m$, and $\nu_{i}(\{0\})=i$. Consider $S=\sum_{i=1}^{\infty} U_{\nu_{i}}^{+}$, acting on $H=\sum_{i=1}^{\infty} \oplus H^{2}\left(\nu_{i}\right)$. No factor of $S$ has even the $C^{*}$-SIP, but we claim that $S$ has the $W^{*}$-SIP. To see this, let $\chi=\chi_{\{0\}}$ be the characteristic function of $\{0\}$; a computation shows that

$$
\sigma\left(T_{\chi}\right)=\{0\} \cup\left(\bigcup_{i=1}^{\infty}\{i /(i+1)\}\right)^{-} .
$$

It follows that if $\Delta$ is any proper Borel subset of $\sigma(N)$ such that $0 \in \Delta$ or if $\Delta=\partial \mathbf{D}$, then $\theta(E(\Delta) K, H)=0$. Lastly, if $\Delta \subseteq \partial \mathbf{D}$ is a proper Borel set such that $E(\Delta)<E(\partial \mathrm{D})$, then it is possible to construct a sequence of functions $\left\{h_{n}\right\} \subseteq$ $H^{2}(m)$ such that $\left\|h_{n}\right\|=1$, for each index $n$, and such that $\int_{\Delta}\left|h_{n}\right|^{2} d m \rightarrow 1$ (reason: $U_{m}^{+}$has the $W^{*}$-SIP). If, for each $n$, we define a function $g_{n}(z)=z h_{n}(z)$, it follows that $\int\left|g_{n}\right|^{2} d \nu_{1}=1$ and that $\int_{\Delta}\left|g_{n}\right|^{2} d \nu_{1} \rightarrow 1$. Letting $f_{n}=\left\{g_{n}, 0,0, \ldots\right\} \in$ $\sum_{i=1}^{\infty} \oplus H^{2}\left(\nu_{i}\right)$ yields a sequence of unit vectors such that $\left\|E(\Delta) f_{n}\right\| \rightarrow 1-$ i.e., that $\theta(E(\Delta) K, H)=0$ in this case as well. Thus, the angle condition is satisfied, for all proper Borel subsets of $\sigma(N)$, and $S$ has the $W^{*}$-SIP.

We should like to close this section with some comments on the distinction between the $C^{*}$ - and $W^{*}$-SIP. If $S$ has the $C^{*}$-SIP, then it follows from Theorem 2.1 that if $\Delta \subseteq \sigma(N)$ is any proper Borel set containing a nonempty, relatively open subset of $\sigma(N)$, then $\theta(E(\Delta) K, H)=0$. If $\Delta \subseteq \sigma(N)$ is a proper Borel subset which does not contain a relatively open subset of $\sigma(N)$, then by regularity it is possible to find a decreasing sequence of relatively open sets $\left\{\Delta_{n}\right\} \subseteq \sigma(N)$ such that $\Delta \subseteq$ $\cap \Delta_{n}$, and such that $E\left(\left(\cap \Delta_{n}\right) \backslash \Delta\right)=0$. Since $\left.P E\left(\Delta_{n}\right)\right|_{H}$ converges in the strong operator topology to $\left.\operatorname{PE}(\Delta)\right|_{H}$, it is not necessarily true that $\left\|\left.P E(\Delta)\right|_{H}\right\|=$ $\lim \left\|\left.P E\left(\Delta_{n}\right)\right|_{H}\right\|=1$, yet this must be the case whenever $S$ has the $W^{*}-$ SIP as well.

Secondly, although Theorem 2.1 states that $1 \in \sigma\left(T_{\chi}\right)$, if $\chi$ is the characteristic function of a nonempty, relatively open subset of $\sigma(N)$, whenever $S$ has the $C^{*}$-SIP, it does not guarantee that $0 \in \sigma\left(T_{\chi}\right)$ as well. In fact, by using the 
regularity of the spectral measure, it is easy to see that the condition that $0 \in \sigma\left(T_{\chi}\right)$, for all characteristic functions associated with relatively open subsets of $\sigma(N)$, is equivalent to the condition that $0 \in \sigma\left(T_{\chi}\right)$, for all characteristic functions associated with proper Borel sets-i.e., that $S$ have the $W^{*}$-SIP.

4. Purity and essential normality. Examples 2.4 and 3.5 suggest that one may construct subnormal operators in a fairly artificial way so as to have the $C^{*}$ - or $W^{*}$-SIP. It may be more reasonable to consider only irreducible subnormal operators-although this may sometimes be too restrictive. A more natural assumption for a subnormal operator is purity.

4.1. LemMA. Suppose that $S$ is pure and has the $C^{*}-S I P\left(W^{*}-S I P\right)$. If $\Delta \subseteq \sigma(N)$ is a nonempty, relatively open (proper Borel) set, then $E(\Delta) K$ is infinite dimensional. Further, $E(\Delta) K \cap H$ is either $(0)$ or infinite dimensional.

Proof. Suppose that $\Delta \subseteq \sigma(N)$ is a relatively open (proper Borel) subset of $\sigma(N)$, and let $M=E(\Delta) K \cap H$. Because $S$ is pure, and because $M$ is an invariant subspace for $S, M$ must be either (0) or infinite dimensional (otherwise $\left.S\right|_{M}$ would be normal). If the latter is the case, then we are done. Otherwise, if $M=(0)$ and if $E(\Delta) K$ is finite dimensional, it would follow that $H+E(\Delta) K$ would be closed. This would imply that $\theta(E(\Delta) K, H)>0$.

4.2. Corollary. If $S$ is pure and has the $C^{*}-S I P\left(W^{*}-S I P\right)$, then $f(N)$ has no isolated eigenvalues of finite multiplicity, for all $f \in C(\sigma(N))\left(L^{\infty}(\mu)\right)$. In any case, $\sigma(f(N))=\sigma_{e}(f(N))$, the essential spectrum of $f(N)$, for all appropriate $f$.

Proof. If $f \in C(\sigma(N))\left(L^{\infty}(\mu)\right)$, then $\lambda$ is an isolated eigenvalue of finite multiplicity for $f(N)$ if and only if $E\left(f^{-1}(\{\lambda\})\right) K$ is finite dimensional-which cannot occur, by Lemma 4.1. The last indicated relationship is well known for normal operators with no isolated eigenvalues of finite multiplicity (see, for example, Fillmore-Stampfli-Williams [1972]).

If $A$ is any operator on $H$, then a scalar $\lambda$ belongs to the reducing essential spectrum of $A$, denoted $R_{e}(A)$, if there exists an orthonormal sequence $\left\{h_{n}\right\} \subseteq H$ such that $\left\|(A-\lambda) h_{n}\right\|+\left\|(A-\lambda)^{*} h_{n}\right\| \rightarrow 0$ (see, for example, Pearcy [1978]). In fact, for purposes of the definition, one need only require that the sequence $\left\{h_{n}\right\}$ be a sequence of unit vectors which converges to zero weakly (see Fillmore-StampfliWilliams [1972]).

4.3. Proposition. If $S$ is pure and has the $C^{*}$-SIP $\left(W^{*}\right.$-SIP), and if $f \in C(\sigma(N))$ $\left(L^{\infty}(\mu)\right)$, then $\sigma(f(N)) \subseteq R_{e}\left(T_{f}\right)$.

Proof. Suppose that $f \in C(\sigma(N))\left(L^{\infty}(\mu)\right)$, and let $\lambda \in \sigma(f(N))$. We claim that there exists a sequence of unit vectors $\left\{h_{n}\right\} \subseteq H$ which converges to zero weakly and for which $\left\|(f(N)-\lambda) h_{n}\right\| \rightarrow 0$. If this is shown, then it will follow that $\left\|\left(T_{f}-\lambda\right) h_{n}\right\|+\left\|\left(T_{f}-\lambda\right)^{*} h_{n}\right\| \leqslant\left\|(f(N)-\lambda) h_{n}\right\|+\left\|(f(N)-\lambda)^{*} h_{n}\right\| \rightarrow 0$, and the result follows.

To see that such a sequence exists, first consider the case when $\lambda \in \sigma(f(N))$ is 
not isolated. Choose a decreasing sequence of relatively open subsets $\left\{\Delta_{n}\right\} \subseteq$ $\sigma(f(N))$ such that $\lambda \in \cap \Delta_{n}, \Delta_{n+1}^{-} \subseteq \Delta_{n}$ for all $n$, and such that diam $\Delta_{n} \rightarrow 0$. Taking a subsequence, if necessary, the sequence $\left\{f^{-1}\left(\Delta_{n} \backslash \Delta_{n+1}^{-}\right)\right\}$is a sequence of relatively open (proper Borel) subsets of $\sigma(N)$; as in the proof of Theorem 2.1, construct a sequence of unit vectors $\left\{h_{n}\right\} \subseteq H$ such that $\left\|E\left(f^{-1}\left(\Delta_{n} \backslash \Delta_{n+1}^{-}\right)\right) h_{n}\right\| \rightarrow$ 1. Necessarily, $h_{n} \rightarrow 0$ in the weak topology, and we are done.

If, however, $\lambda$ is an isolated point in $\sigma(f(N))$, then let $M=E\left(f^{-1}(\{\lambda\})\right) K \cap H$. By Lemma 4.1, $M$ is either (0) or infinite dimensional. In the latter case, it is an easy matter to produce the desired sequence. But if $M=(0)$, then let $\left\{h_{n}\right\}$ be any sequence of unit vectors in $H$ such that $\left\|E\left(f^{-1}(\{\lambda\})\right) h_{n}\right\| \rightarrow 1$ and such that $\left\|(f(N)-\lambda) h_{n}\right\| \rightarrow 0$. The unit ball of $H$ is weakly compact, so we may assume that there exists $h \in H$ for which $\|h\| \leqslant 1$ and for which $h_{n} \rightarrow h$ weakly. If $\Delta=$ $\sigma(f(N)) \backslash\{\lambda\}$, then $E\left(f^{-1}(\Delta)\right) h_{n}$ converges to $E\left(f^{-1}(\Delta)\right) h$ weakly; thus, $\left\|E\left(f^{-1}(\Delta)\right) h\right\| \leqslant \lim \left\|E\left(f^{-1}(\Delta)\right) h_{n}\right\|=0$. Thus, $h \in M$, and so $h=0$. The sequence $\left\{h_{n}\right\}$ is then as desired.

4.4. Corollary. If $S$ is pure and has the $C^{*}$-SIP ( $\left.W^{*}-S I P\right)$, then the operators $\left\{T_{f}: f \in C(\sigma(N))\right\}\left(\left\{T_{f}: f \in L^{\infty}(\mu)\right\}\right)$ are extremely noncompact. That is, if $C$ is a compact operator and $f$ is in the appropriate algebra, then $\left\|T_{f}+C\right\|>\left\|T_{f}\right\|$. In particular, when $S$ is pure, the only function $f$ for which $T_{f}$ is compact is the zero function.

Proof. If $\pi$ is the canonical projection onto the Calkin algebra, then

$$
\begin{aligned}
\left\|T_{f}+C\right\| & \geqslant\left\|\pi\left(T_{f}+C\right)\right\|=\left\|\pi\left(T_{f}\right)\right\| \\
& \geqslant \sup \left\{|\lambda|: \lambda \in \sigma\left(\pi\left(T_{f}\right)\right)\right\} \geqslant \sup \left\{|\lambda|: \lambda \in R_{e}\left(T_{f}\right)\right\} \\
& \geqslant \sup \{|\lambda|: \lambda \in \sigma(f(N))\}=\|f(N)\|=\left\|T_{f}\right\|,
\end{aligned}
$$

where the last inequality has followed from Proposition 4.3.

We remark that the notion of extremely noncompact operator is due to Coburn, who established the above result when $S$ is the unilateral shift (Coburn [1966]).

4.5. Corollary. Let $S$ be a pure subnormal operator with either the $C^{*}$ - or $W^{*}$-SIP. If $T_{f}$ is Fredholm, for $f$ in the appropriate algebra, then $f$ is invertible (in the same algebra).

Lastly, let us suppose that the subnormal operator $S$ is essentially normal-that is to say, that $S$ has compact self-commutator $S^{*} S-S S^{*}$. This is equivalent to supposing that $P N(1-P): H^{\perp} \rightarrow H$ is compact. If $p=p(\bar{z}, z)$ is any polynomial in the complex variables $\bar{z}$ and $z$, then $P p\left(N^{*}, N\right)(1-P)$ and $(1-P) p\left(N^{*}, N\right) P$ will be compact operators. It follows that if $f \in C(\sigma(N))$, then $P f(N)(1-P)$ and $(1-P) f(N) P$ are compact.

4.6. Proposition. Let $S$ be pure, essentially normal, and have the $C^{*}$-SIP. Then

(i) for all $f \in C(\sigma(N)), T_{f}$ is essentially normal;

(ii) if $f, g \in C(\sigma(N))$, then $T_{f} T_{g}-T_{f g}$ is compact; 
(iii) the mapping $f \rightarrow \pi\left(T_{f}\right)$ defines $a^{*}$-isometric isomorphism of $C(\sigma(N))$ onto $C^{*}(\pi(S))$, thus inducing $a^{*}$-isometric isomorphism of $C^{*}(N)$ onto $C^{*}(\pi(S))$;

(iv) $T_{f}$ is Fredholm if, and only if, $f$ is invertible, for all $f \in C(\sigma(N))$.

Proof. The first condition follows from the comments preceding the proposition and the equation $T_{f}^{*} T_{f}-T_{f} T_{f}^{*}=P f(N)(1-P) f(N)^{*} P-P f(N)^{*}(1-P) f(N) P$. The second condition is immediate from the relation $T_{f g}-T_{f} T_{g}=$ $P f(N)(1-P) g(N) P$. Condition (iii) now follows from (ii) and Corollary 4.4. The last condition now follows from condition (iii).

4.7. Corollary. If $S$ is irreducible, essentially normal, and has the $C^{*}-S I P$, then $C^{*}(S)=\left\{T_{f}+C: f \in C(\sigma(N))\right.$ and $C$ is compact $\}$. In particular, this describes $C^{*}\left(U_{m}^{+}\right)$.

Proof. Under the above assumptions, $\left\{T_{f}+C: f \in C(\sigma(N))\right.$ and $C$ is compact $\}$ is a selfadjoint algebra containing $S$ (Proposition 4.6(ii)). Further, Corollary 4.4 implies that it is closed-i.e., that it is a $C^{*}$-algebra. Since $C^{*}(S)$ is a $C^{*}$-algebra containing $S$ and the compact operators, the desired equality results.

\section{REFERENCES}

M. B. Abrahamse and R. G. Douglas, [1976], A class of subnormal operators related to multiply connected domains, Adv. in Math. 19, 106-148.

J. W. Bunce and J. A. Deddens, [1977], On the normal spectrum of a subnormal operator, Proc. Amer. Math. Soc. 63, 107-110.

L. A. Coburn, [1966], Weyl's theorem for non-normal operators, Michigan Math. J. 13, 285-288.

R. G. Douglas, [1972], Banach algebra techniques in operator theory, Academic Press, New York.

R. G. Douglas and Carl M. Pearcy, [1965], Spectral theory of generalized Toeplitz operators, Trans. Amer. Math. Soc. 115, 433-444.

P. A. Fillmore, J. G. Stampfli and James P. Williams, [1972], On the essential numerical range, the essential spectrum, and a problem of Halmos, Acta Sci. Math. (Szeged) 33, 179-192.

Richard Frankfurt, [1975], Subnormal weighted shifts and related function spaces, J. Math. Anal. Appl. 52, 471-489.

P. R. Halmos, [1951], Introduction to Hilbert space and the theory of spectral multiplicity, Chelsea, New York.

, [1952], Spectra and spectral manifolds, Ann. Soc. Math. Polon. 25, 43-49.

[1967], A Hilbert space problem book, Van Nostrand, Princeton, N.J.

[1970], Ten problems in Hilbert space, Bull. Amer. Math. Soc. 76. 887-933.

P. Hartman and A. Wintner, [1950], On the spectra of Toeplitz's matrices, Amer. J. Math. 72, 359-366.

Robert F. Olin, [1976], Functional relationships between a subnormal operator and its minimal normal extension, Pacific J. Math. 63, 221-229.

Carl M. Pearcy, [1978], Some recent developments in operator theory, CBMS Regional Conf. Series in Math., Amer. Math. Soc., Providence, R.I.

Allen L. Shields, [1974], Weighted shift operators and analytic function theory, in Topics in Operator Theory, edited by C. Pearcy, Math. Surveys, no. 13, Amer. Math. Soc., Providence, R. I.

Department of Mathematics, Boston College, Chestnut Hill, Massachusetts 02167 